\title{
INNER AND OUTER FUNCTIONS ON RIEMANN SURFACES
}

\author{
MICHAEL VOICHICK ${ }^{1}$ AND LAWRENCE ZALCMAN
}

1. Introduction. In this paper we generalize to Riemann surfaces the factorization theory for functions in the Hardy classes, $H^{p}$, on the unit disk.

Let $R$ be a region on a Riemann surface with boundary $\Gamma$ consisting of a finite number of simple closed analytic curves such that $R \cup \Gamma$ is compact and $R$ lies on one side of $\Gamma$. For $1 \leqq p<\infty H^{p}(R)$ is the class of functions $F$ analytic on $R$ such that $|F|^{p}$ has a harmonic majorant. $H^{\infty}(R)$ is the class of bounded analytic functions on $R$. The above classes are the usual generalizations of the Hardy classes on the disk (cf. [4], [5], and [6]). However, to obtain a factorization of these functions which closely parallels the factorization on the disk we are led to more general classes of functions. To this end, we say that a (multiple-valued) analytic function $F$ on $R$ is multiplicative if $|F|$ is single-valued and define $M H^{p}(R), 1 \leqq p<\infty$, to be the class of multiplicative analytic functions $F$ on $R$ such that $|F|^{p}$ has a harmonic majorant. Also, we define $M H^{\infty}(R)$ to be the class of bounded multiplicative functions on $R$.

Let $d \mu$ be the harmonic measure on $\Gamma$ with respect to some fixed point $t_{0} \in R$. If $F \in M H^{p}$ then $|F|$ has nontangential boundary values $\left|F^{*}\right|$ a.e. $[d \mu]$ on $\Gamma$. Moreover, $\left|F^{*}\right| \in L^{p}(\Gamma, d \mu)$ and $\log \left|F^{*}\right|$ $\in L^{1}(\Gamma, d \mu)$ if $F \not \equiv 0$. These facts follow easily from the corresponding results on the disk. (Cf. [10, p. 496].)

We say $F \in M H^{p}(R)$ is an outer function if

$$
\log \left|F\left(t_{0}\right)\right|=\int_{\mathrm{r}} \log \left|F^{*}\right| d \mu .
$$

$\Phi \in M H^{\infty}(R)$ is an inner function if $|\Phi *|=1$ a.e. on $\Gamma$. A nonvanishing inner function is said to be a singular function. An inner function, $B$, is said to be a Blaschke product if

$$
|B(t)|=\exp \left[-\sum_{k} p_{k} G\left(t, t_{k}\right)\right]
$$

for all $t \in R$, where $G$ is the Green's function for $R,\left\{t_{k}\right\}$ is a sequence of points on $R$, and $\left\{p_{k}\right\}$ is a sequence of non-negative integers. When

Received by the editors December 28, 1964.

${ }^{1}$ Partially supported by the Carnegie Corporation. 
$R$ is the unit disk, the definitions above are equivalent to the classical ones.

Theorem 1. Let $F \in M H^{p}(R)$. Then $|F|=\left|B\|S\| F_{1}\right|$ where $B$ is a Blaschke product, $S$ is a singular function, and $F_{1}$ is an outer function in $M H^{p}(R)$. These factors are unique up to multiplicative constants of modulus one.

Since $H^{p}(R) \subset M H^{p}(R)$, this theorem subsumes a factorization of functions in $H^{p}(R)$. However, the factors of a single-valued function in $H^{p}(R)$ need not be single-valued.

Theorem 1 is stated in terms of the moduli of functions since there is no natural way to define the product of multiplicative functions; that is, the product would depend on the branches chosen.

In our proof we shall make use of the factorization on the disk. A convenient reference for this is [3].

It should be remarked that results related to ours are in [7] for $R$ an annulus and in [2] for the general case.

2. Proof of Theorem 1. Let $K=\{z \cup z \mid<1\}$ and $T: K \rightarrow R$ be a universal covering map of $R$. Let $Q=\{q\}$ be the group of fractional linear transformations such that $T \circ q=T$. A function $f$, analytic on $K$, is said to be modulus invariant if $|f \circ q|=|f|$ for all $q \in Q$. It is easy to see that $f$ is modulus invariant on $K$ if, and only if, $f \circ T^{-1}$ is multiplicative on $R$. Also, for $f$ modulus invariant, $f \in H^{p}(K)$ if, and only if, $f \circ T^{-1} \in M H^{p}(R)$. For $F \in M H^{p}(R)$ and $f \circ T^{-1}=F$ let $f=b s f_{1}$ where $b$ is a Blaschke product, $s$ is a singular function, and $f_{1}$ is an outer function in $H^{p}(K)$. Then $f$ is modulus invariant and by Lemma 4.6 in [10] bs and $f_{1}$ are also modulus invariant.

LEMMA. $b$ is modulus invariant.

Proof. First observe that if $z$ is a zero of $f$ then $q(z)$ is a zero of $f$ with the same multiplicity for each $q \in Q$. Thus $b \circ q / b \in H^{\infty}(K)$ for each $q \in Q$ since $b$ and $b \circ q$ have the same zeros with the same multiplicities. Then for all $q \in Q b / b \circ q=\left(b \circ q^{-1} \circ q\right) /(b \circ q) \in H^{\infty}(K)$. It follows that $|b|=|b \circ q|$ for all $q \in Q$.

Let $\Delta$ be the fundamental domain of $Q$ and $E$ the union of the free sides of $\Delta$; that is, $E=\bar{\Delta} \cap\{|z|=1\}$. Then the harmonic measure $d \mu$ corresponds to the measure

$$
d m(\theta)=\frac{1}{2 \pi}\left[\sum_{q \in Q} \frac{1-\left|q\left(z_{0}\right)\right|^{2}}{\left|e^{i \theta}-q\left(z_{0}\right)\right|^{2}}\right] d \theta
$$

on $E$ where $z_{0} \in \Delta$ and $T\left(z_{0}\right)=t_{0}$. (Cf. [9, pp. 526, 529].) Moreover, $d m(\theta)$ and $d \theta$ are mutually absolutely continuous. 
Let $B=b \circ T^{-1}, S=s \circ T^{-1}$, and $F_{1}=f_{1} \circ T^{-1}$. Then $|F|$ $=|B||S|\left|F_{1}\right|$. Since $b$ is an inner function and $s$ is a singular function, it is immediate by virtue of the relation between $d \theta$ and $d m(\theta)$ that $B$ is an inner function and $S$ is a singular function. (Cf. [10, Lemma 4.4].) It remains to show that $B$ is a Blaschke product and $F_{1}$ is an outer function.

We shall consider $F_{1}$ first. Since $d m(\theta)$ corresponds to $d \mu$, it follows that

$$
\int_{\Gamma} \log \left|F_{1}^{*}\right| d \mu=\int_{E} \log \left|f_{1}^{*}\right| d m(\theta) .
$$

Now since the set $U_{q \in Q} q(E)$ is of full measure on $|z|=1$ (see [9, p. 525]) and $\left|f_{1}^{*} \circ q\right|=\left|f_{1}^{*}\right|$ a.e. on $|z|=1$, a change of variable for the integral on the right (see [9, pp. 526-528]) yields

$$
\begin{aligned}
\int_{\Gamma} \log \left|F_{1}^{*}\right| d \mu & =\int_{E} \log \left|f_{1}^{*}\right| d m(\theta) \\
& =\frac{1}{2 \pi} \int_{0}^{2 \pi} \log \left|f_{1}^{*}\right| \frac{1-\left|z_{0}\right|^{2}}{\left|e^{i \theta}-z_{0}\right|} d \theta \\
& =\log \left|f_{1}\left(z_{0}\right)\right|=\log \left|F_{1}\left(t_{0}\right)\right| .
\end{aligned}
$$

Therefore $F_{1}$ is an outer function.

Next we show that $B$ is a Blaschke product. Let $\left\{t_{k}\right\}$ be the set of zeros of $F$ and $p_{k}$ be the multiplicity of the zero at $t_{k}$. For each $t_{k}$ let $z_{k}$ be a point in $K$ such that $T\left(z_{k}\right)=t_{k}$. Then for $q \in Q, b$ has a zero at $q\left(z_{k}\right)$ of multiplicity $p_{k}$. All the zeros of $b$ occur in this way. Thus,

$$
|b(z)|=\prod_{k} \prod_{q \in Q}\left|\frac{q\left(z_{k}\right)-z}{1-\bar{q}\left(z_{k}\right) z}\right|^{p_{k}} .
$$

Now for $z, z^{\prime} \in K$

$$
G\left(T(z), T\left(z^{\prime}\right)\right)=\sum_{q \in Q} \log \left|\frac{1-\bar{q}\left(z^{\prime}\right) z}{z-q\left(z^{\prime}\right)}\right| .
$$

(See [9, p. 529].) Hence for $t=T(z)$

$$
|B(t)|=|b(z)|=\prod_{k} \exp \left[-p_{k} G\left(t, t_{k}\right)\right]=\exp \left[-\sum_{k} p_{k} G\left(t, t_{k}\right)\right]
$$

Thus $B$ is a Blaschke product and $F=|B||S|\left|F_{1}\right|$ is the desired factorization. Since $b, s$, and $f_{1}$ are unique up to multiplicative constants of modulus one, the same is true for $B, S$, and $F_{1}$. This completes the proof of Theorem 1 . 
3. Closed invariant subspaces of $H^{2}(R)$. Let $A(R)$ be the class of (single-valued) functions continuous on $\bar{R}$ and analytic on $R$. A closed subspace $V$ of $H^{2}(R)$ is said to be invariant if $F V \subset V$ for all $F \in A(R)$. For $\Phi$ an inner function on $R$ let $V(\Phi)=\left\{F \in H^{2}(R) \mid\right.$ $|F|^{2} /|\Phi|^{2}$ has a harmonic majorant on $\left.R\right\}$. In $[10$, Theorem 8.11] it is shown that $V(\Phi)$ is a closed invariant subspace of $H^{2}(R)$. The following theorem, which reduces to a well-known result of Beurling's [1, Theorem IV, p. 253] for the case $R=K$, is proved in [10, Theorem 2].

TheOREM 2. If $V$ is a closed invariant subspace of $H^{2}(R)$ then there is an inner function $\Phi$ such that $V=V(\Phi)$.

By Theorem 1 we have for $F \in H^{p}(R),|F|=|\Phi|\left|F_{1}\right|$ where $\Phi$ is an inner function. We call $\Phi$ an inner factor of $F$.

Theorem 3. Let $F \in H^{2}(R)$ and $V[F]$ be the smallest closed invariant subspace of $H^{2}(R)$ which contains $F$. Then $V[F]=V(\Phi)$ where $\Phi$ is an inner factor of $F$.

Proof. Clearly $V(\Phi) \supset V[F]$. By Theorem 2, $V[F]=V\left(\Phi_{0}\right)$ for some inner function $\Phi_{0}$. Let $H \in V(\Phi)$. We must show $H \in V\left(\Phi_{0}\right)$. For $f=F \circ T$ and $h=H \circ T$ let $f=\phi f_{1}$ and $h=\psi h_{1}$ be the inner-outer factorizations of $f$ and $h$ respectively such that $\Phi=\phi \circ T^{-1}$. Then $\Psi=\psi \circ T^{-1}$ is an inner factor of $H$. Let $\phi_{0}$ be a modulus invariant inner function such that $\Phi_{0}=\phi_{0} \circ T^{-1}$. Since $F \in V\left(\Phi_{0}\right),|F|^{2} /\left|\Phi_{0}\right|^{2}$ has a harmonic majorant; and it follows that $f / \phi_{0} \in H^{2}(K)$. This implies $\phi / \phi_{0}$ is an inner function. By a similar argument $\psi / \phi$ is an inner function. Thus $\psi / \phi_{0}=(\psi / \phi)\left(\phi / \phi_{0}\right)$ is an inner function. Hence $|\Psi| /\left|\Phi_{0}\right|$ is bounded. This implies $H \in V\left(\Phi_{0}\right)$.

Corollary 1. Let $F \in H^{2}(R)$. Then $V[F]=H^{2}(R)$ if, and only if, $F$ is an outer function. (Cf. [7, Theorem 1, p. 128].)

The following result was proved by D. Sarason ([7, pp. 112, 128] and $[8$, Theorem 4, p. 596]). We offer a different proof.

Corollary 2. Let $R=\{z|r<| z \mid<1\}$ and suppose $F \in H^{2}(R)$. Then $V[F]=H^{2}(R)$ if, and only if, for $0 \leqq \delta \leqq 1$

$$
\begin{aligned}
\int_{0}^{2 \pi} \log \left|F\left(r^{\delta} e^{i t}\right)\right| d t= & (1-\delta) \int_{0}^{2 \pi} \log \left|F^{*}\left(e^{i t}\right)\right| d t \\
& +\delta \int_{0}^{2 \pi} \log \left|F^{*}\left(r e^{i t}\right)\right| d t
\end{aligned}
$$

Proof. By virtue of Corollary 1 it is sufficient to show that $F$ satisfies $\left({ }^{*}\right)$ if, and only if, $F$ is an outer function. Suppose $F$ is an outer function, then 


$$
\begin{aligned}
\int_{0}^{2 \pi} \log \left|F\left(r^{\delta} e^{i t}\right)\right| d t & =\int_{0}^{2 \pi} \frac{1}{2 \pi} \int_{\partial R} \log \left|F^{*}(w)\right| \frac{\partial G\left(w, r^{\delta} e^{i t}\right)}{\partial n}|d w| d t \\
& =\int_{|w|=1} \log \left|F^{*}(w)\right| \frac{1}{2 \pi} \int_{0}^{2 \pi} \frac{\partial G\left(w, r^{\delta} e^{i t}\right)}{\partial n} d t|d w| \\
& +\int_{|w|=r} \log \left|F^{*}(w)\right| \frac{1}{2 \pi} \int_{0}^{2 \pi} \frac{\partial G\left(w, r^{\delta} e^{i t}\right)}{\partial n} d t|d w| \\
& =I_{1}(\delta)+I_{2}(\delta) .
\end{aligned}
$$

Now $\partial G\left(e^{i \theta}, r^{\delta} e^{i t}\right) / \partial n=\partial G\left(e^{-i t}, r^{\delta} e^{-i \theta}\right) / \partial n$. Thus for $|w|=1$

$$
\begin{aligned}
\frac{1}{2 \pi} \int_{0}^{2 \pi} \frac{\partial G\left(w, r^{\delta} e^{i t}\right)}{\partial n} d t & =\frac{1}{2 \pi} \int_{0}^{2 \pi} \frac{\partial G\left(e^{i t}, r^{\delta} \bar{w}\right)}{\partial n} d t \\
& =\text { harmonic measure of }\{|z|=1\} \text { at } r^{\delta} \bar{w} \\
& =1-\left(\log r^{\delta} / \log r\right)=1-\delta .
\end{aligned}
$$

Thus $I_{1}(\delta)=(1-\delta) \int_{0}^{2 \pi} \log \left|F^{*}\left(e^{i t}\right)\right| d t$. A similar argument shows $I_{2}(\delta)$ $=\delta \int_{0}^{2 \pi} \log \left|F^{*}\left(r e^{i t}\right)\right| d t$. Hence $F$ satisfies $\left(^{*}\right)$.

To complete the proof we show that if $F$ is not an outer function then $\left({ }^{*}\right)$ is not satisfied. Let $F_{1}$ be an outer factor of $F$. Then $|F|$ $\left\langle\left|F_{1}\right|\right.$ on $R$ and $|F|=\left|F_{1}\right|$ a.e. on $\partial R$. We have shown that $F_{1}$ satisfies $\left(^{*}\right)$. Thus $\left(^{*}\right)$ does not hold for $F$.

\section{REFERENCES}

1. A. Beurling, On two problems concerning linear transformations in Hilbert space, Acta Math. 81 (1949), 239-255.

2. M. Hasumi, Invariant subspace theorems for finite Riemann surfaces, ditto copy, 1964.

3. K. Hoffman, Banach spaces of analytic functions, Prentice-Hall, Englewood Cliffs, N. J., 1962.

4. M. Parreau, Sur les moyennes des fonctions harmoniques et analytiques et la classification des surfaces Riemann, Ann. Inst. Fourier (Grenoble) 3 (1951), 103-197.

5. H. L. Royden, The boundary values of analytic and harmonic functions, Math. Z. 78 (1962), 1-24.

6. W. Rudin, Analytic functions of class $H_{p}$, Trans. Amer. Math. Soc. 78 (1955), $46-66$.

7. D. Sarason, The $H^{p}$ spaces of annuli, Dissertation, Univ. of Michigan, Ann Arbor, Mich., 1963.

8. - Doubly invariant subspaces of annulus operators, Bull. Amer. Math. Soc. 69 (1963), 593-596.

9. M. Tsuji, Potential theory in modern function theory, Maruzen, Tokyo, 1959.

10. M. Voichick, Ideals and invariant subspaces of analytic functions, Trans. Amer. Math. Soc. 111 (1964), 493-512.

\section{Dartmouth College,}

UNIVERSITY OF WISCONSIN AND

Massachusetrs INSTITUTE of TechNoLogy 